Bei der Darstellung dieses Salzes scheint sich anfangs stets Quecksilberrhodanid und metallisches Quecksilber zu bilden. Ist die Flüssigkeit hinreichend sauer, so wird der graue oder schwarze Niederschlag durch längeres Stehen weiss, was darauf beruht, dass Quecksilberrhodanid und salpetersaures Quecksilberoxydul sich in unlösliches Rhodanür und salpetersaures Quecksilberoxyd umsetzen: $\mathrm{Hg}(\mathrm{CNS})^{2}+\mathrm{Hg}^{2} \mathrm{~N}^{2} \mathrm{O}^{6}=\mathrm{Hg}^{2}(\mathrm{CNS})^{2}+\mathrm{HgN}^{2} \mathrm{O}^{6}$.

\title{
Analyse der Glimmer von Utö und Faston und Bemerkungen über die Zusammensetzung der Kaliglimmer überhaupt; \\ von
}
C. Rammelsberg in Berlin*).

Keine der grossen und wichtigen Mineralgruppen bietet in krystallographischer, optischer und chemischer Hinsicht so viel Eigenthümliches und zum Theil Unerklärbares, wie die Glimmer. Ihre Structur und ihre meist wenig messbaren Krystalle liessen sie lange für sechsgliedrig halten; eine gut krystallisirte Abänderung (vom Vesuv) wurde als zwei - und eingliedrig erkannt, später für zweigliedrig-partialfächig erklärt, bis sich zeigte, dass ihre Form geometrisch in aller Strenge eben so wohl sechsgliedrig, als zweigliedrig oder zwei- und eingliedrig gelten könne.

Uebrigens ist neuerlich die angebliche zweigliedrige Partialfächigkeit durch vollständigere Beobachtungen widerlegt (Hessen berg).

In optischer Beziehung uuterschied man lange einund zweiaxige Glimmer. Allein man nimmt jetzt gewöhnlich an, dass die anscheinend einaxigen solche sind, deren beide Axen einen sehr kleinen Winkel machen, da man

*) Als Abdruck aus der Zeitschrift der deutschen geologischen Gesellschaft, Jahrg. 1866, vom Herrn Verfasser übergeben. 
gefunden hat, dass optisoh zweiaxige Blättchen, in einer um $90^{\circ}$ gekreuzten Stellung auf einander gelegt, so dass die Ebenen ihrer optischen Axen sich gleicher Art schneiden, die Erscheinungen optisch einaxiger Krystalle zeigen.

Aber nicht allein ist der Winkel der optischen Axen bei den Glimmern ein äusserst veränderlicher, von $0^{0}$ bis $77^{0}$ gehend, obwohl die Mittellinie immer senkrecht zur Spaltungsfläche steht und negativ ist, sondern die Ebene der optischen Axen ist bei manchen Glimmern senkrecht gegen diejenige anderer. Die Untersuchungen lasser erkennen, dass solche verschiedene Glimmer, verschieden in der Grösse des Winkels und in der Lage der Fbene der optischen Axen, an einem Fundorte vorkommen (Warwick).

Unwillkürlich erinnern diese Verhältnisse der Glimmer an die von Scacchi zur Sprache gebrachten Fälle von Polysymmetrie. Das zweigliedrige, optisch zweiaxige schwefelsaure Kali ist geometrisch gleich dem schwefelsauren Kalinatron, welches sechsgliedrig und optisch einaxig ist. Wenn dies beweist, dass die künstlichen $\mathrm{Ab}$ theilungen, welche wir den Symmetriegesetzen der Krystalle anpassen - unsere Krystallsysteme -, dem Reichthume der Erscheinungen nicht Genüge leisten, so müssen die Glimmer besonders zu einem weiteren Studium anregen, und es wäre wohl denkbar, dass es unter ihnen auch wahre optisch einaxige gäbe.

Die chemische Unterscheidung der Glimmer erfolgt vorlüufig am besten nach der Natur der sogenannten starken Basen, welche die Analyse aus ihnen darstellt. Denn finden wir auch manche derselben in allen Glimmern wieder, so tritt doch eine in der Regel bei einer ganzen Abtheilung als herrschend hervor.

Alkaliglimmer nenne ich daher solche, welche durch ein Alkali charakterisirt sind. Unter ihnen sind die wichtigsten die Kaliglimmer von heller Farbe, 46-50 Proc. Kieselsäure und im Mittel 10 Proc. Kali gebend, neben ihm nur wenig Magnesia und höchstens 
8 Proc. Eisenoxyd. Viele scheinen nur Spuren von Natron, einige bis 5 Proc. desselben zu enthalten. Fluor ist wohl, wenn auch nur in kleiner Menge, doch wahrscheinlich in allen enthalten und vom Wasser, glaube ich, gilt dasselbe. Der Winkel ihrer optischen Axen ist gross.

Die Natronglimmer (Paragonit), feinschuppige, helle Glimmer, sind bis jetzt wenig bekannt. Ausser Natron, dem stets Kali beigesellt ist, sind kaum andere starke Basen darin enthalten.

Die Lithionglimmer, optisch den Kaliglimmern gleich, enthalten neben vorherrschendem Kali auch Lithion und Natron und sind durch ihren hohen Fluorgehalt und ihre Schmelzbarkeit ausgezeichnet. Theils eisenfrei (Lepidolith), theils eisenhaltig, entbehren sie aller anderen starken Basen fast ganz.

Vor Kurzem habe ich zwei Kaliglimmer untersucht, den goldgelben von Utö, den H. Rose vor 50 Jahren in Berzelius' Laboratorium analysirte bei Gelegenheit der Arbeit, welche ihn zur Entdeckung des Fluors in den Glimmern führte. Ich wünschte zu wissen, in wie weit die Fortschritte der Mineralanalyse bei einer Wiederholung Aenderungen des früheren Resultats bewirken können, was insbesondere für Fluor, Wasser und die Alkalien in Frage kommt.

Der zweite ist hellbräunlicher, in dünnen Blättchen farbloser Glimmer, der, von Orthoklas und Quarz begleitet, in grossen sechsseitigen Prismen zu Easton in Pensylvanien vorkommt.

Das Volumengewicht des Glimmers von Utö ist $=$ 2,836, das von Easton $=2,904$, und das Resultat der Analysen, wobei ich H. Rose's beifüge, ist:

Utö

Easton

H. Rose

\begin{tabular}{|c|c|c|c|}
\hline Wasser & 2,30 & 2,50 & 3,36 \\
\hline Fluor. & 0,96 & 1,32 & 1,05 \\
\hline Kieselsäure... & 47,50 & 45,75 & 46,74 \\
\hline Thonerde. & 37,20 & 35,48 & 35,10 \\
\hline
\end{tabular}




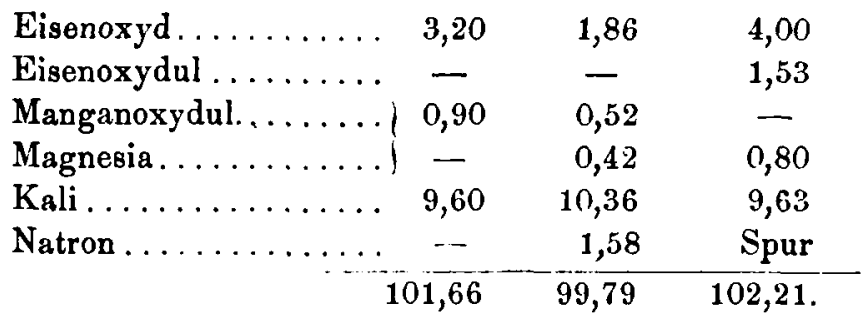

Der Glimmer von Utö enthält so wenig Eisen, dass eine besondere Prüfung auf die Oxyde desselben nicht nöthig ist. Was zunächst den Glimmer von Utö betrifft, so stimmen H. Rose's und meine Analyse in dem Verhältnisse der Kieselsäure und Thonerde sehr genau überein. Es ist nämlich

$$
\begin{aligned}
\mathrm{Al}^{2}: \mathrm{Si} & =1: 2,18 \text { At. bei H. Rose, } \\
& =1: 2,20 \text { At. bei mir. }
\end{aligned}
$$

Auch wenn das sämmtliche Eisen als Eisenoxyd vorausgesetzt und sein Aequivalent dem $\mathrm{Al}^{2}$ hinzugerechnet wird, bleibt das Verhältniss ziemlich unverändert, trotzdem H. Rose fast doppelt so viel Eisen $(2,24$ Proc.) fand als ich (1,3 Proc.); es wird nämlich:

$$
\begin{aligned}
\left(\mathrm{Al}^{2}, \mathrm{Fe}^{2}\right): \mathrm{Si} & =1: 2,07 \mathrm{H} . \mathrm{R} . \\
& =1: 2,13 \mathrm{Rg} .
\end{aligned}
$$

Anders aber gestaltet sich das Verhältniss des Kaliums zu jenen beiden Elementen. Denn jenes ist bei H. Rose $=7,97$, bei mir aber, mit Zurechnung des Natriumäquivalentes, $=10,60, \mathrm{~d} . \mathrm{h}$. ich habe $4 / 3 \mathrm{mal}$ so viel gefunden als $H$. Rose. Auch wird diese Differenz nicht ausgeglichen durch die kleinen Mengen Mangan und Magnesium, welche bei mir $=1,39$, bei $H$. Rose nur $=0,9$ sind. Daher kommt es, dass das Atomenverhältniss $\mathrm{K}$ ( $\mathrm{Na}, \mathrm{Mg}$, $\mathrm{Mn}): \mathrm{Al}^{2}$ oder $\mathrm{Si}$ in beiden Analysen nicht unerheblich differirt. Es ist nämlich :

$$
\begin{aligned}
\mathrm{K}: \mathrm{Al}^{2},\left(\mathrm{Fe}^{2}\right) & =1: 1,70 \mathrm{~K}: \mathrm{Si}=1: 3,5 \mathrm{H} . \mathrm{R} . \\
& =1: 1,16
\end{aligned}
$$

Wird das Eisen als Oxydul berechnet oder, richtiger gesagt, als zweiwerthig dem Mangan und Magnesium 
zugetheilt, so ist nach seiner Verwandlung in das Kaliumäquivalent:

$$
\begin{aligned}
& \mathrm{K}(\mathrm{Fe}): \mathrm{Al}^{2}=1: 1,18 \quad \mathrm{~K}(\mathrm{Fe}): \mathrm{Si}=1: 2,6 \mathrm{H} . \mathrm{R} \text {. } \\
& =1: 2,5 \quad=1: 5,5 \mathrm{Rg} \text {. }
\end{aligned}
$$

In der früheren Art in Sauerstoffverhältnissen ausgedrückt, würden diese Berechnungen geben: Sauerstoff von

(H. Rose)

$\mathrm{RO}: \mathrm{R}^{2} \mathrm{O}^{3}=1: 9,6$

$\mathrm{R}^{2} \mathrm{O}^{3}: \mathrm{SiO} 2=1: 1,38$

$\mathrm{RO}: \mathrm{SiO}^{2}=1: 13,2$

$\mathrm{RO}, \mathrm{R}^{2} \mathrm{O}^{3}: \mathrm{SiO}^{2}=1: 1,25$

(Rammelsberg)

also:

$$
\mathrm{RO}: \mathrm{R}^{2} \mathrm{O}^{3}: \mathrm{SiO}^{2}=1: 9,6: \begin{aligned}
& 13,2 \\
& \lceil 13,3
\end{aligned}
$$$$
1: 7,0
$$$$
1: 1,42
$$$$
1: 10
$$$$
1: 1,24
$$

Oder, wenn das Fisen lediglich als Oxydul berechnet wird:

(H. Rose)

$$
\mathrm{RO}: \mathrm{Al}^{2} \mathrm{O}^{3}=1: 7
$$$$
\mathrm{Al}^{2} \mathrm{O}^{3}: \mathrm{SiO}^{2}==1: 1,45
$$$$
\mathrm{RO}: \mathrm{SiO}^{2}=1: 10
$$

$\mathrm{RO}, \mathrm{Al}^{2} \mathrm{O}^{3}: \mathrm{SiO}^{2}=1: 1,27$ also:

$$
\mathrm{RO}: \mathrm{Al}^{2} \mathrm{O}^{3}: \mathrm{SiO}^{2}=1: 7:\left\{\begin{array}{l}
10 \\
10,15
\end{array}\right.
$$

(Rammelsberg)

$$
\begin{array}{ll}
1 & : 5,9 \\
1 & : 1,5 \\
1 & : 8,65 \\
1 & : 1,26
\end{array}
$$

Bei diesen Berechnungen ist aber auf das $W$ as ser keine Rücksicht genommen. H. Rose hatte bereits das Wasser als chemisch gebundenes bezeichnet, und ich habe mich überzeugt, dass die Glimmer, nachdem sie bei einer dem Glühen nahen Temperatur erhalten worden, in starker Hitze oft eine bedeutende Menge Wasser liefern, welches von Fluorkiesel oder vielmehr Kieselsäure und Kieselfluorwasserstoffaäure begleitet ist. Bei dem Glimmer von Utö betrug dieser Verlust 4,3 Proc. ${ }^{*}$ ). Rechnet man dio

*) Die Angaben älterer Analysen lassen sich schwer corrigiren. H. Rose fand im Glimmer von Utö 0,53 Proc. Flusssäure und 2,63 Wasser. Diese Zahlen wïren in 0,96 und 2,3 zu verwandeln. 
dem gefundenen Fluorgehalte entsprechende Menge Fluorkiesel ab, so bleiben 2,3 Proc. Wasser.

Den neueren Ansichten zufolge ist der Wasserstoff des Wassers ein Vertreter des gleich ihm einwerthigen Kaliums; er muss folglich bei der Berechnung diesem zugefügt werden. Thut man dies bei den beiden von mir untersuchten Glimmern, so werden die Atomverhältnisse viel einfacher wie sonst.

Atomverhältnisse von

$$
\mathrm{H} \quad: \mathrm{K}: \mathrm{Al} \mathbf{l}^{2}: \mathrm{Si} \quad \mathrm{H}, \mathrm{K}: \mathrm{Al}^{2}: \mathrm{Si}
$$

$$
\begin{aligned}
& \text { Utö... } \left.=0,79^{*}\right): 0,86: 1: 2,13=1,65: 1: 2,13 \\
& \text { Easton }=1,0^{*} \text { ) }: 0,8: 1: 2,12=1,8: 1: 2,12 .
\end{aligned}
$$

Mit einer kleinen Correction für die am schwersten genau bestimmbaren Elemente II und $\mathrm{K}$ sind also nicht allein beide Glimmer gleich, sondern auch höchst einfach zusammengesetzt, denn das Atomrerhältniss $2: 1: 2$ giebt, wenn $\mathrm{H}=\mathrm{K}$,

$$
\underset{\mathrm{Si}^{2}}{\mathrm{~K} 12} \underset{\mathrm{O}}{\mathrm{H}} \mathrm{O}^{8} \text {, entsprechend } 2 \mathrm{H}^{4} \mathrm{SiO}^{4} \text {. }
$$

Mit der Analyse der Glimmer von Aschaffenburg und von Gossen beschäftigt, hoffe jch später über die chemische Constitution der Kaliglimmer mehr sagen zu können, will aber schon jetzt bemerken, dass die Glimmer von Utö und Easton mit der Mehrzahl aller anderen 1 Atom $\mathrm{Al}^{2}$ $\left(\mathrm{Fe}^{2}\right)$ gegen 2 Atome $\mathrm{Si}$, eine Minderzahl $1: 3$ Atome enthalten, und dass in jener ersten Abtheilung auf 1 Atom $\mathrm{Al}^{2}\left(\mathrm{Fe}^{2}\right)$ stets 2 Atome der einwerthigen Elemente, $\mathrm{K}$ und H, kommen.

Verwandelt man in der eben entwickelten Formel die 2 Atome einwerthiger Elemente ( $K$ und $H$ ) in ihr Aequivalent, d. h. in 1 Atom eines zweiwerthigen, z. B. Magnesium, so erhält man $\mathrm{Mg} \mathrm{Al}{ }^{2} \mathrm{Si}^{2} \mathrm{O}^{8}$. Beide Formeln drücken die Zusammensetzung von Singulosilikaten aus.

*) Diese Zahlen sind in der Wirklichkeit sicher grösser, weil der geglühte Glimmer nicht alles Fluor verloren bat. 
Nun habe ich längst zu zeigen gesucht*), dass die Magnesiaglimmer Singulosilikate sind. Die vorhergehenden Betrachtungen lehren, dass auch die untersuchten und noch viele andere (vielleicht alle) Kaliglimmer Singulosilikate sind. Es ist meines Wissens dies der erste auf factischen Grundlagen ruhende Schritt, die Analogie der Zusammensetzung für beide Glimmerarten zu erweisen.

\section{Zur Methode der Aschenanalyse; \\ von \\ Prof. Dr. E. Reichardt, in Jena**).}

Die Ermittelung der anorganischen Bestandtheile in pflanzlichen oder thierischen Substanzen ist schon seit lange der vielfachsten Erörterungen theilhaftig geworden. Die Wichtigkeit dieser Stoffe für den pflanzlichen oder thierischen Organismus, für die Erhaltung des Lebens, wie für den Aufbau der einzelnen Theile ist sicher nicht zu unterschätzen, wie es früher lange Zeit geschehen war. Die kleinsten Mengen einzelner Aschenbestandtheile treten so constant auf, dass dadurch schon allein ihre Unentbehrlichkeit genügend erwiesen wird. Dies die Veranlassung einer besonderen Besprechung der Ausführung der Aschenanalysen, um gleichzeitig mit Vereinfachung die möglichste Genauigkeit der Methode zu bieten.

Die Darstellung einer Asche durch Verbrennung der verbrennbaren Theile bietet um so mehr Schwierigkeiten, je mehr von phosphorsauren Salzen oder von Kieselerde, auch Thonerde, in den organischen Substanzen vorhanden ist und besonders auch, je mehr stickstoff haltige organische Verbindungen zugegen sind. Mannigfache Vorschläge

*) Handbuch der Mineral-Chemie, S. 669.

**) Als Separatabdruck aus der Jenaischen Zeitschrift für Medicin und Naturwiss. IV. Bd. 1867 von Hra. Verfasser erhalten.

D. Red. 